\title{
Black hole mass estimation using a relation between the BLR size and emission line luminosity of AGN
}

\begin{abstract}
X.-B. Wu, R. Wang, M. Z. Kong, F. K. Liu, and J. L. Han
National Astronomical Observatories of Chinese Academy of Sciences and Department of Astronomy, Peking University, Beijing 100871, China

e-mail: wuxb@bac.pku.edu.cn

Received 11 December 2003 / Accepted 3 March 2004

Abstract. An empirical relation between the broad line region (BLR) size and optical continuum luminosity is often adopted to estimate the BLR size and then the black hole mass of AGNs. However, optical luminosity may not be a good indicator of photoionizing luminosity for extremely radio-loud AGNs because the jets usually contribute significantly to the optical continuum. Therefore, the black hole masses derived for blazar-type AGNs with this method are probably overestimated. Here we first derived a tight empirical relation between the BLR size and the $\mathrm{H}_{\beta}$ emission line luminosity, $R$ (light - days $)=$ $24.05\left(L_{\mathrm{H}_{\beta}} / 10^{42} \mathrm{ergs} \mathrm{s}^{-1}\right)^{0.68}$, from a sample of $34 \mathrm{AGNs}$ with the BLR size estimated with the reverberation mapping technique. Then we applied this relation to estimate the black hole masses of some AGNs and found that for many extremely radioloud AGNs the black hole masses obtained with the $R-L_{\mathrm{H}_{\beta}}$ relation are systematically lower than those derived previously with the $R-L_{5100} \AA$ relation, while for radio-quiet and slightly radio-loud AGNs the results obtained with these two methods are almost the same. The difference of black hole masses estimated with these two relations increases with the radio-loudness for extremely radio-loud AGNs, which is consistent with the fact that their equivalent widths of the $\mathrm{H}_{\beta}$ emission line become smaller at greater radio-loudness. If the small $\mathrm{H}_{\beta}$ equivalent widths of extremely radio-loud AGNs are indeed caused by the beaming effect, we argue that the optical emission line luminosity may be a better tracer of ionizing luminosity for blazartype AGNs and the black hole masses derived with the $R-L_{\mathrm{H}_{\beta}}$ relation are probably more accurate.
\end{abstract}

Key words. black hole physics - galaxies: active - galaxies: nuclei - quasars: general - quasars: emission lines

\section{Introduction}

Supermassive black holes are essential for AGN activity (Lynden-Bell 1969; Rees 1984). The black hole masses of some nearby AGNs have recently been estimated by the reverberation mapping technique (Wandel et al. 1999; Ho 1999; Kaspi et al. 2000), with which the size of the broad line region (BLR) can be measured from the time delay between the flux variations of the continuum and the emission lines of AGNs. The black hole mass is then estimated using the Virial theorem from the BLR size and the characteristic velocity (determined by the full width at half-maximum (FWHM) of the emission line). So far, reverberation studies have yielded the black hole masses of about 20 Seyfert 1 galaxies and 17 nearby bright quasars.

An empirical relation between the BLR size $(R)$ and the optical continuum luminosity at $5100 \AA\left(L_{5100} \AA\right)$ has been derived by Kaspi et al. (2000) using the observed data of 34 nearby AGNs. Because the measurement of the BLR size with the reverberation mapping technique needs a long-term monitoring of continuum and emission line fluxes, it is impractical for most AGNs. Therefore, the empirical relation has been frequently adopted to estimate the BLR size and then to derive the black hole masses for AGNs in some samples of mostly radio-quiet objects (Laor 2000; McLure \& Dunlop 2001; Wandel 2002), and of purely radio-loud objects (Lacy et al. 2001; Gu et al. 2001; Oshlack et al. 2002). However, the optical luminosity of some radio-loud AGNs (especially blazars) may not be a good indicator of ionizing luminosity, which is usually related to the UV/optical radiation from the accretion disk around the central black hole. The relativistic jets of blazar-type AGNs not only dominate the radio and high energy X-ray and $\gamma$ ray radiation, but also significantly contribute to the optical luminosity in some cases (Scarpa \& Urry 2002). For example, many optical jets have been discovered recently in AGNs by the HST (Scarpa et al. 1999; Jester 2003; Parma et al. 2003), which clearly suggests that the jets contribute significantly in the optical band. Furthermore, optical synchrotron radiation has been detected for many other radio-loud AGNs (Whiting et al. 2001; Chiaberge et al. 2002; Cheung et al. 2003). Therefore, the measured optical continuum luminosity of some extremely radio-loud AGNs is significantly contributed by the optical radiation from the jets and may be much larger than the ionizing luminosity required to produce broad emission lines. Using the empirical relation between the BLR size and optical luminosity at $5100 \AA$, which 
was obtained based on the sample of mostly radio-quiet AGNs (Kaspi et al. 2000), one would significantly overestimate the actual BLR size and hence the black hole mass of these radioloud AGNs. Oshlack et al. (2002) have shown that their estimated black hole masses would be lower if the synchrotron contribution to the optical flux is subtracted. However, it is not easy to make such a correction for a large sample of radioloud AGNs. In addition, the contribution of the host galaxy to the optical continuum should also be taken into account especially when the host galaxy of AGNs can be resolved optically. Therefore, optical luminosity may not be a good indicator of photoionization luminosity of AGNs in some cases.

In this paper we will first derive an empirical relation between the BLR size and the $\mathrm{H}_{\beta}$ emission line luminosity for 34 AGNs in the sample of reverberation mapping studies. We then argue that the BLR size obtained from the $\mathrm{H}_{\beta}$ luminosity is more reasonable at least for some extremely radioloud AGNs. Finally we apply this new empirical relation to estimate the black hole masses of some quasars and compare them with previous results.

\section{The relation between the BLR size and $\mathrm{H}_{\beta}$ luminosity}

Kaspi et al. (2000) have compiled the observational data of 17 Seyfert galaxies (Wandel et al. 1999) and 17 nearby quasars with black hole masses estimated with the reverberation mapping technique. Using a linear fit to the available data with errors, they got an empirical relation between the BLR size and the optical continuum luminosity at $5100 \AA$ as:

$$
\begin{aligned}
R_{\mathrm{BLR}}(\text { light }- \text { days })= & \left(32.9_{-1.9}^{+2.0}\right) \\
& \times\left[L_{5100 \AA} / 10^{44} \mathrm{ergs} \mathrm{s}^{-1}\right]^{0.700 \pm 0.033} .
\end{aligned}
$$

With an ordinary least square (OLS) bisector method (Isobe et al. 1990), we can obtain such a relation as $R_{\mathrm{BLR}}$ (light - days) $=31.1\left[L_{5100 \AA} / 10^{44} \mathrm{ergs} \mathrm{s}^{-1}\right]^{0.701}$, which is almost the same as that shown above. We should keep in mind that this $R-L_{5100 \AA}$ relation was obtained with mostly radio-quiet AGNs. The optical continuum luminosity may not be a good indicator of photoionizing luminosity for some extremely radio-loud AGNs. Instead, the emission line luminosity may be a better indicator because it is free of the beaming effects of the jet.

Using the available data of BLR sizes and $\mathrm{H}_{\beta}$ fluxes for 34 AGNs in the reverberation mapping studies, we can investigate the relation between the BLR size and the $\mathrm{H}_{\beta}$ emission line luminosity (including both broad and narrow components). In Table 1 we listed the BLR size and the luminosity data of these 34 AGNs. The $\mathrm{H}_{\beta}$ luminosity is calculated from the $\mathrm{H}_{\beta}$ flux which is available for 16 PG quasars, 8 AGNs in the Ohio sample, and another 9 Seyfert 1 galaxies (see references listed in Table 1). Because there is no available data of $\mathrm{H}_{\beta}$ flux for PG 1351+640 (Kaspi et al. 2000), we exclude this object from our investigation. In addition, we add another Seyfert galaxy, Mrk 279, to our sample because both the BLR size and the $\mathrm{H}_{\beta}$ flux have been measured recently (Santos-Lleo et al. 2001). All $\mathrm{H}_{\beta}$ luminosity data have been corrected for Galactic extinction using the values from NED 1 (see also Burstein \& Heiles 1982). The cosmology with Hubble constant $H_{0}=75 \mathrm{~km} \mathrm{~s}^{-1} \mathrm{Mpc}^{-1}$ and deceleration parameter $q_{0}=0.5$ were adopted through out the paper.

With these data, we derive an empirical relation between the BLR size and $\mathrm{H}_{\beta}$ luminosity. With the OLS bisector method we obtained:

$$
\begin{aligned}
& \log R(\text { light }- \text { days })=(1.381 \pm 0.080) \\
& \quad+(0.684 \pm 0.106) \log \left(L_{\mathrm{H}_{\beta}} / 10^{42} \mathrm{ergs} \mathrm{s}^{-1}\right) .
\end{aligned}
$$

The slope of this relation is slightly flatter than that of $R-$

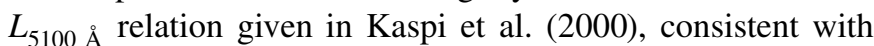
$L_{\mathrm{H}_{\beta}} \propto L_{B}^{0.93}$ obtained by Ho \& Peng (2001) for PG quasars. The Spearman's rank correlation coefficient of our $R-L_{\mathrm{H}_{\beta}}$ relation is 0.91 , slightly higher than 0.83 for the $R-L_{5100 \AA}$ relation (Kaspi et al. 2000), which implies that the $R-L_{\mathrm{H}_{\beta}}$ relation is slightly tighter than the $R-L_{5100} \AA$ relation. We also used the linear fit to the data with errors that was used by Kaspi et al. (2000) to derive the $R-L_{5100 \AA}$ relation. Applying this method to the $R-L_{\mathrm{H}_{\beta}}$ relation yields the same result as the OLS bisector method. We also noticed that a similar relation between the BLR size and $\mathrm{H}_{\alpha}$ luminosity has been mentioned recently by Wang \& Zhang (2003).

In Fig. 1 we show the dependence of the BLR size on $L_{\mathrm{H}_{\beta}}$ and $L_{5100 \AA}$. The two relations are similar and thus the $R-L_{\mathrm{H}_{\beta}}$ relation can be an alternative to the $R-L_{5100} \AA$ relation in estimating the BLR size for radio-quiet AGNs.

\section{Comparison of black hole mass estimation of AGNs from two relations}

Since the $R-L_{5100} \AA$ relation obtained by Kaspi et al. (2000) has been frequently used to estimate the BLR size and the black hole mass of both radio-quiet and radio-loud AGNs, it is important to investigate the applicability of such an approach for radio-loud objects.

Brotherton (1996) studied the emission line properties of 59 radio-loud quasars. We adopted his published values of absolute $V$-band magnitude, equivalent width and FWHM of the $\mathrm{H}_{\beta}$ emission line. The continuum luminosity at $5100 \AA$ and the $\mathrm{H}_{\beta}$ luminosity (scaled to our cosmology parameters) were calculated after considering the Galactic extinction and K-correction (optical spectral index was assumed to be 0.3). We then estimated the BLR size using both $R-L_{\mathrm{H}_{\beta}}$ and $R-L_{5100 \AA}$ relations and derived the black hole mass with the formula $M_{\mathrm{BH}}=3 V_{\mathrm{FWHM}}^{2} R / 4 G$ (here we assumed the BLR velocity $V \sim(\sqrt{3} / 2) V_{\text {FWHM }}$ as in Kaspi et al. 2000). With these two relations, we also estimated the black hole masses of another 27 radio-loud quasars with available data of both the equivalent width and FWHM of $\mathrm{H}_{\beta}$ emission line in the Parkes Half-Jansky Flat-spectrum Sample (PHFS) (Drinkwater et al. 1997; Francis et al. 2000; Oshlack et al. 2001). We compared the black hole masses obtained with the $R-L_{\mathrm{H}_{\beta}}$ and $R-L_{5100 \AA}$ relations in Fig. 2. Evidently the masses obtained

\footnotetext{
1 http://nedwww.ipac.caltech.edu
} 
Table 1. The BLR size and luminosity data of 34 AGNs in the reverberation mapping studies.

\begin{tabular}{|c|c|c|c|c|c|c|}
\hline Name & $z$ & $A_{B}$ & $\begin{array}{c}R_{\mathrm{BLR}} \\
\text { (light-days) }\end{array}$ & $\begin{array}{c}L_{5100 \AA} \\
\left(10^{42} \mathrm{erg} / \mathrm{s}\right)\end{array}$ & $\begin{array}{c}L_{\mathrm{H}_{\beta}} \\
\left(10^{42} \mathrm{erg} / \mathrm{s}\right)\end{array}$ & Ref. \\
\hline $3 \mathrm{C} 120$ & 0.033 & 0.570 & $42.0_{-20.0}^{+27.0}$ & $73.00 \pm 13.00$ & $1.222 \pm 0.120$ & 1 \\
\hline 3C 390.3 & 0.056 & 0.170 & $22.9_{-8.0}^{+6.3}$ & $64.00 \pm 11.00$ & $1.433 \pm 0.171$ & 3 \\
\hline Akn 120 & 0.033 & 0.400 & $37.4_{-6.3}^{+5.1}$ & $139.00 \pm 26.00$ & $2.496 \pm 0.463$ & 1 \\
\hline F 9 & 0.046 & 0.000 & $16.3_{-7.6}^{+3.3}$ & $137.00 \pm 15.00$ & $2.056 \pm 0.174$ & 4 \\
\hline IC $4329 A$ & 0.016 & 0.000 & $1.4_{-2.9}^{+3.3}$ & $16.40 \pm 2.10$ & $0.183 \pm 0.014$ & 5 \\
\hline Mrk 110 & 0.035 & 0.000 & $18.8_{-6.6}^{+6.3}$ & $38.00 \pm 13.00$ & $0.811 \pm 0.203$ & 1 \\
\hline Mrk 279 & 0.030 & 0.000 & $16.2_{-5.4}^{+5.1}$ & $66.00 \pm 6.30$ & $1.023 \pm 0.071$ & 12 \\
\hline Mrk 335 & 0.026 & 0.100 & $16.4_{-3.2}^{+5.1}$ & $62.20 \pm 5.70$ & $1.160 \pm 0.073$ & 1 \\
\hline Mrk 509 & 0.034 & 0.180 & $76.7_{-6.0}^{+6.3}$ & $147.00 \pm 15.00$ & $3.070 \pm 0.332$ & 1 \\
\hline Mrk 590 & 0.026 & 0.050 & $20.0_{-2.9}^{+4.4}$ & $51.00 \pm 9.60$ & $0.498 \pm 0.143$ & 1 \\
\hline Mrk 79 & 0.022 & 0.230 & $17.7_{-8.4}^{+4.8}$ & $42.30 \pm 5.60$ & $0.619 \pm 0.041$ & 1 \\
\hline Mrk 817 & 0.031 & 0.000 & $15.0_{-3.4}^{+4.2}$ & $52.60 \pm 7.70$ & $0.740 \pm 0.123$ & 1 \\
\hline NGC 3227 & 0.004 & 0.020 & $10.9_{-10.9}^{+5.6}$ & $2.02 \pm 0.11$ & $0.017 \pm 0.002$ & 6 \\
\hline NGC 3783 & 0.010 & 0.470 & $4.5_{-3.1}^{+3.6}$ & $17.70 \pm 1.50$ & $0.292 \pm 0.021$ & 7 \\
\hline NGC 4051 & 0.002 & 0.000 & $6.5_{-4.1}^{+6.6}$ & $0.525 \pm 0.03$ & $0.0048 \pm 0.0005$ & 8 \\
\hline NGC 4151 & 0.003 & 0.000 & $3.0_{-1.4}^{+1.8}$ & $7.20 \pm 0.42$ & $0.152 \pm 0.010$ & 9 \\
\hline NGC 5548 & 0.017 & 0.000 & $21.2_{-0.7}^{+2.4}$ & $27.00 \pm 5.30$ & $0.421 \pm 0.092$ & 10 \\
\hline NGC 7469 & 0.016 & 0.120 & $4.9_{-1.1}^{+0.6}$ & $55.30 \pm 1.60$ & $0.423 \pm 0.019$ & 11 \\
\hline PG 0026+129 & 0.142 & 0.130 & $113.0_{-21.0}^{+18.0}$ & $700.00 \pm 100.00$ & $5.693 \pm 0.493$ & 2 \\
\hline PG $0052+251$ & 0.155 & 0.120 & $134.0_{-23.0}^{+31.0}$ & $650.00 \pm 110.00$ & $9.595 \pm 1.119$ & 2 \\
\hline PG 0804+761 & 0.100 & 0.110 & $156.0_{-13.0}^{+15.0}$ & $660.00 \pm 120.00$ & $13.95 \pm 0.908$ & 2 \\
\hline PG 0844+349 & 0.064 & 0.080 & $24.2_{-9.1}^{+10.0}$ & $172.00 \pm 17.00$ & $2.585 \pm 0.257$ & 2 \\
\hline PG $0953+414$ & 0.239 & 0.000 & $151.0_{-27.0}^{+22.0}$ & $1190.00 \pm 160.00$ & $19.39 \pm 1.129$ & 2 \\
\hline PG $1211+143$ & 0.085 & 0.130 & $101.0_{-29.0}^{+23.0}$ & $493.00 \pm 80.00$ & $8.588 \pm 1.056$ & 2 \\
\hline PG $1226+023$ & 0.158 & 0.000 & $387.0_{-50.0}^{+58.0}$ & $6440.00 \pm 770.00$ & $88.37 \pm 7.192$ & 2 \\
\hline PG 1229+204 & 0.064 & 0.000 & $50.0_{-23.0}^{+24.0}$ & $94.00 \pm 10.0$ & $1.601 \pm 0.202$ & 2 \\
\hline PG $1307+085$ & 0.155 & 0.020 & $124.0_{-80.0}^{+45.0}$ & $527.00 \pm 52.00$ & $9.603 \pm 1.301$ & 2 \\
\hline PG 1411+442 & 0.089 & 0.000 & $102.0_{-37.0}^{+38.0}$ & $325.00 \pm 28.00$ & $5.268 \pm 0.285$ & 2 \\
\hline PG 1426+015 & 0.086 & 0.120 & $95.0_{-39.0}^{+31.0}$ & $409.00 \pm 63.00$ & $4.952 \pm 0.465$ & 2 \\
\hline PG $1613+658$ & 0.129 & 0.040 & $39.0_{-14.0}^{+20.0}$ & $696.00 \pm 87.00$ & $7.014 \pm 0.451$ & 2 \\
\hline PG $1617+175$ & 0.114 & 0.150 & $85.0_{-25.0}^{+19.0}$ & $237.00 \pm 41.00$ & $4.060 \pm 0.493$ & 2 \\
\hline PG $1700+518$ & 0.292 & 0.020 & $88.0_{-182.0}^{+190.0}$ & $2710.00 \pm 190.00$ & $35.29 \pm 1.851$ & 2 \\
\hline PG 1704+608 & 0.371 & 0.000 & $319.0_{-285.0}^{+184.0}$ & $3560.00 \pm 520.00$ & $9.752 \pm 1.257$ & 2 \\
\hline PG 2130+099 & 0.061 & 0.170 & $200.0_{-18.0}^{+67.0}$ & $216.00 \pm 20.00$ & $4.241 \pm 0.381$ & 2 \\
\hline
\end{tabular}

Notes: The Galactic extinction values are adopted from NED. Data of $R_{\mathrm{BLR}}$ and $L_{5100 \AA}$ are taken from Kaspi et al. (2000). The $\mathrm{H}_{\beta}$ luminosity is calculated from the flux given in the literature (see the column "ref"). References: (1) Peterson et al. 1998; (2) Kaspi et al. 2000; (3) Dietrich et al. (1998); (4) Santos-Lleo et al. (1997); (5) Winge et al. (1996); (6) Winge et al. (1995); (7) Strirpe et al. (1994); (8) Peterson et al. (2000); (9) Kaspi et al. (1996); (10) Peterson et al. (2002); (11) Collier et al. (1996); (12) Santos-Lleo et al. (2001).

with the $R-L_{\mathrm{H}_{\beta}}$ relation are systematically lower that those obtained with the $R-L_{5100} \AA$ relation for some extremely radioloud quasars.

In Fig. 2 we also show how the difference of black hole masses obtained with these two relations varies with the radioloudness for these two samples of radio-loud quasars. The values of radio-loudness of 27 PHFS quasars were taken from Oshlack et al. (2001) and those of 59 quasars in Brotherton (1996) were calculated from the $R_{V}$ value (defined as the ratio of core radio luminosity and the $V$-band optical luminosity) and the core and extended radio luminosity values listed in his Table 1. It is clear that the difference of black hole masses is small when the radio-loudness is small but becomes larger as the radio-loudness increases. The Spearman's rank correlation coefficient is -0.34 , implying a modest correlation between the difference of black hole masses and the radio-loudness. For some individual quasars with higher radio-loudness, the black hole mass estimated with the $R-L_{5100} \AA$ relation can 

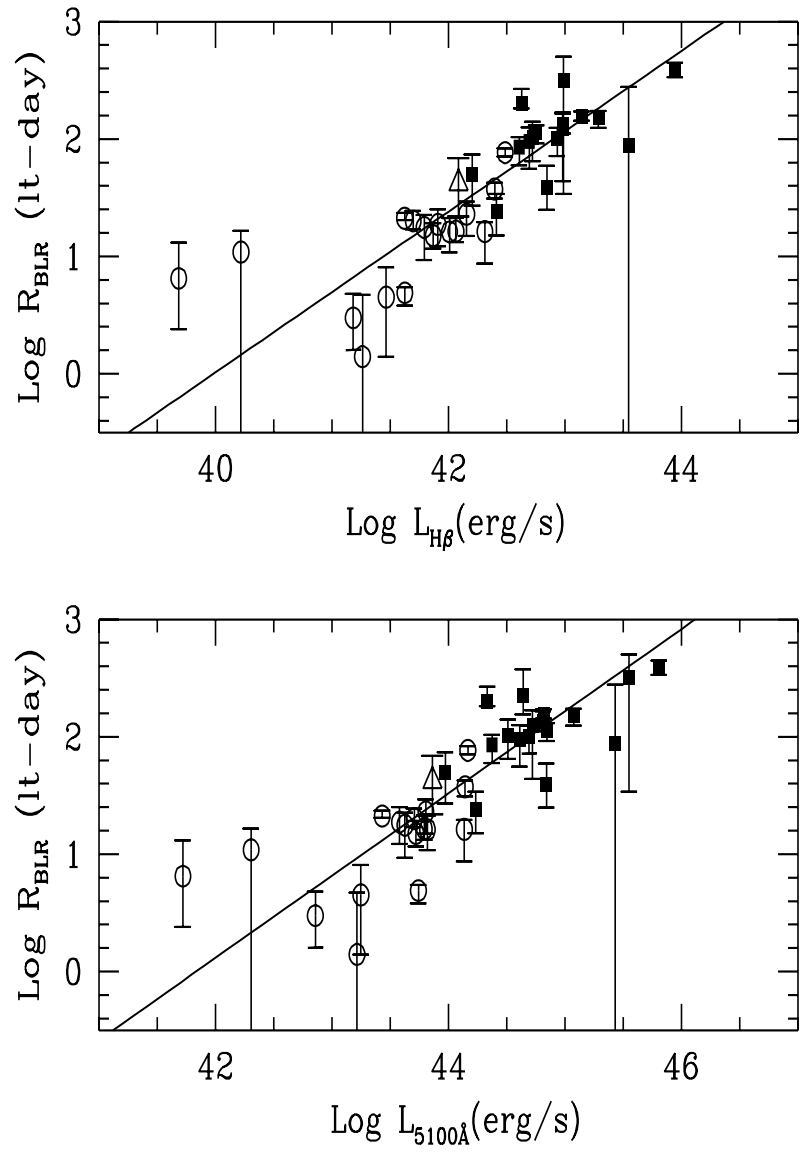

Fig. 1. The relations of BLR size and luminosity for 34 AGNs in the reverberation mapping studies. The open and filled symbols denote Seyfert galaxies and quasars respectively. The upper panel shows the $R-L_{\mathrm{H}_{\beta}}$ relation. The line shows the OLS bisector fit to the data. The lower panel shows the $R-L_{5100} \AA$ relation, which is identical to the Fig. 6 in Kaspi et al. (2000) except that we include Mrk 279 (shown as open triangle). The line represents the linear fit given in Kaspi et al. (2000).

be $3 \sim 10$ times larger than that estimated with the $R-L_{\mathrm{H}_{\beta}}$ relation. In Fig. 3 we also plotted the equivalent width $(E W)$ of the $\mathrm{H}_{\beta}$ emission line against the radio-loudness for objects in these two radio-loud AGN samples. We can see that the $E W\left(\mathrm{H}_{\beta}\right)$ becomes smaller at higher radio-loudness. Such a relation, although with only a modest Spearman's rank correlation coefficient of -0.33 , indicates that the smaller $E W\left(\mathrm{H}_{\beta}\right)$ of some extremely radio-loud AGNs could be at least partly due to beaming effects.

For radio-quiet AGNs, however, both the optical continuum and emission line luminosities are probably free of jet contributions and therefore both can be good tracers of photoionization luminosity. We check this by using the data of 70 low-redshift radio-quiet quasars in the Palomar-Green survey. The emission line properties of these quasars have been studied by Boroson $\&$ Green (1992). The continuum luminosity at $5100 \AA$ and the $\mathrm{H}_{\beta}$ luminosity were estimated from the absolute $V$-band magnitude $\left(M_{V}\right)$ and the equivalent width of $\mathrm{H}_{\beta}$ emission line listed in their Tables 1 and 2 . Using the $R-L_{\mathrm{H}_{\beta}}$ and $R-L_{5100 \AA}$ relations we estimated the BLR sizes and black hole masses of these radio-quiet quasars. The results from the two relations are
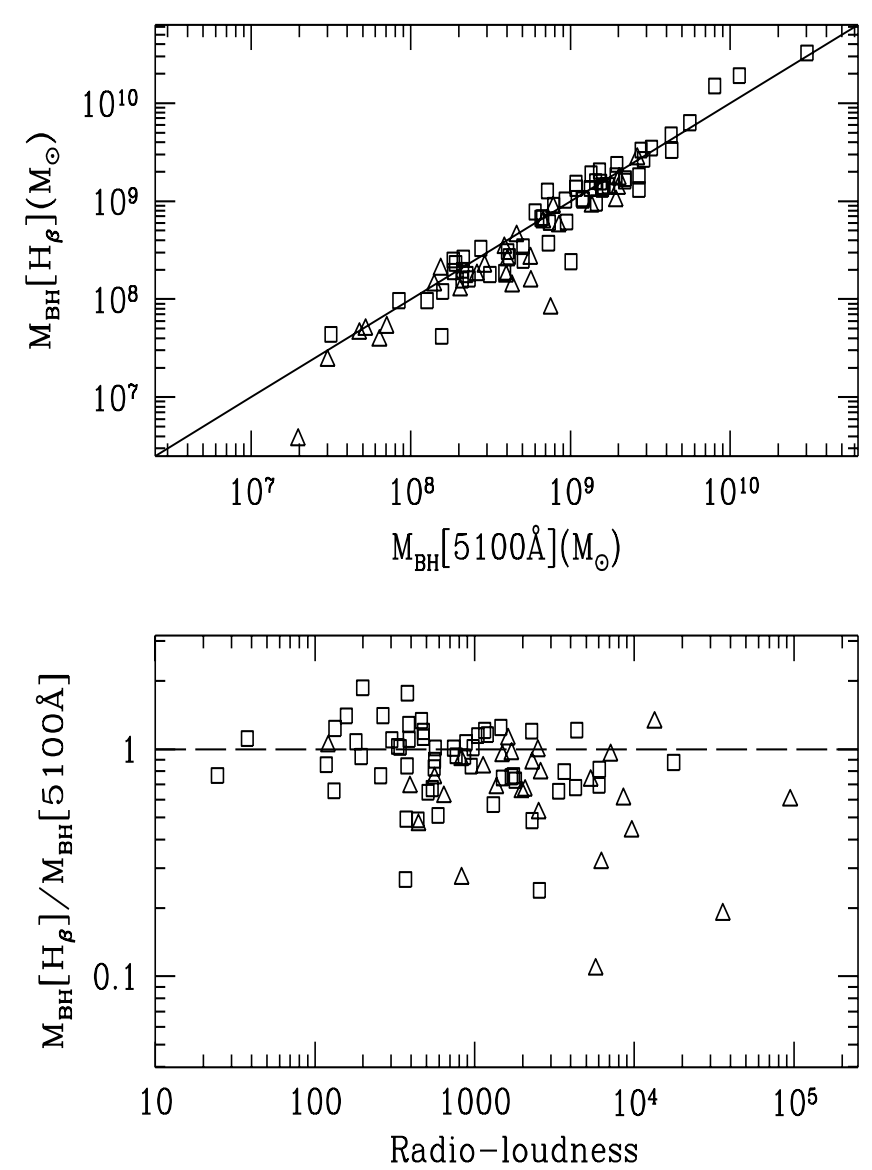

Fig. 2. Upper panel: comparison of the black hole masses of radioloud quasars estimated with the $R-L_{\mathrm{H}_{\beta}}$ and $R-L_{5100 \AA}$ relations. The squares represent 59 quasars in Brotherton (1996) and the triangles represent 27 PHFS quasars in Oshlack et al. (2001). The diagonal line shows the relation where both masses are identical. Lower panel: the ratios of black hole masses estimated with two different relations are plotted against the radio-loudness of radio-loud quasars. The dashed line indicates the case where the two black hole mass estimates are identical. Evidently $M_{\mathrm{BH}}[5100 \AA]$ becomes systematically larger than $M_{\mathrm{BH}}\left[\mathrm{H}_{\beta}\right]$ at higher radio-loudness.

almost identical (see Fig. 4). This is also indicated by the normalized $\chi^{2}$ value of the deviation of the points plotted in the upper panel of Fig. 4, which is 0.94 , much smaller than the value 2.58 for the points plotted in the upper panel of Fig. 2 for radio-loud AGNs. From the lower panel of Fig. 4, we can also see that the difference of the two black hole mass estimates does not correlated with the radio-loudness for radioquiet quasars. The Spearman's rank test gives a correlation coefficient of only 0.05 , much smaller than that for radioloud AGNs.

\section{Discussions}

Using the empirical relation between the BLR size and optical continuum luminosity possibly induces an overestimation of the BLR size and hence the black hole mass of some extremely radio-loud AGNs because of the jet contribution to the optical luminosity. We derived another empirical relation between the BLR size and the emission line luminosity, and demonstrated 


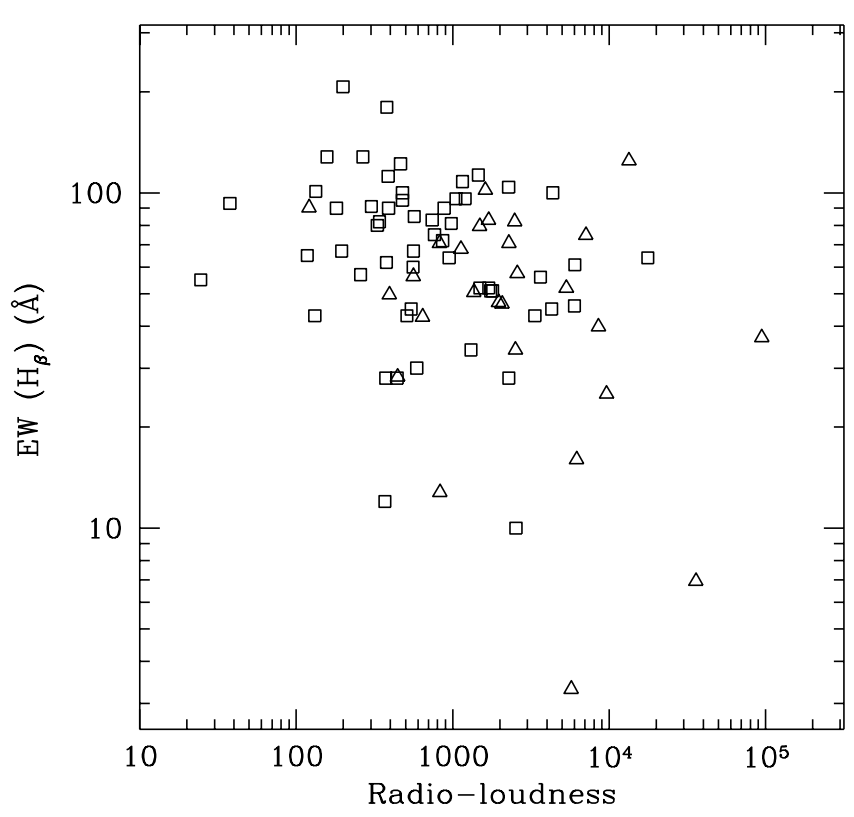

Fig. 3. The relation between the equivalent width of $\mathrm{H}_{\beta}$ emission line and the radio-loudness of radio-loud quasars. The symbols have the same meaning as in Fig. 2.

that it can be used to estimate the BLR size and black hole mass of both radio-quiet and radio-loud AGNs. If the relativistic jets and host galaxy have significant contributions to the optical continuum, the emission line luminosity is probably a better tracer of ionizing luminosity. Comparisons of the estimated black hole masses with these two different empirical relations clearly indicate that the difference becomes significant if the radio-loudness of AGNs is larger. Using the $R-L_{\mathrm{H}_{\beta}}$ relation may result in more accurate estimations of black hole masses of some blazar-type AGNs.

In our study we focused on the possible effects of beaming on the optical continuum in radio-loud AGNs and ignored the difference in BLR physics between radio-loud and radioquiet AGNs. The modest correlation between the $E W\left(\mathrm{H}_{\beta}\right)$ and the radio-loudness may indicate the presence of beaming effects, though some other effects such as a lower covering factor of the BLR of radio-loud AGNs can also lead to smaller $E W\left(\mathrm{H}_{\beta}\right)$ values. Because currently we know little about the difference in the BLR physics between radio-loud and radioquiet AGNs, to prove the validity of our approach it is necessary to compare our estimated black hole mass with an independent estimate, for example, from the correlations of black hole mass with central velocity dispersion and host galaxy luminosity. Unfortunately, not many measured values of central velocity dispersion or host galaxy luminosity for extremely radio-loud AGNs are available. Although there are 8 objects in the sample of Brotherton (1996) with measured host magnitude (McLure \& Dunlop 2001), the radio-loudness of these objects are mostly smaller than 1000 and thus the difference estimated with the $R-L_{\mathrm{H}_{\beta}}$ and $R-L_{5100 \AA}$ relations is rather small. The velocity dispersion measurements for radio-loud quasars are not available and the [OIII] profile in radio-loud AGNs should not be adopted to estimate the central velocity dispersion because of its complexity. Therefore, further imaging studies on the host
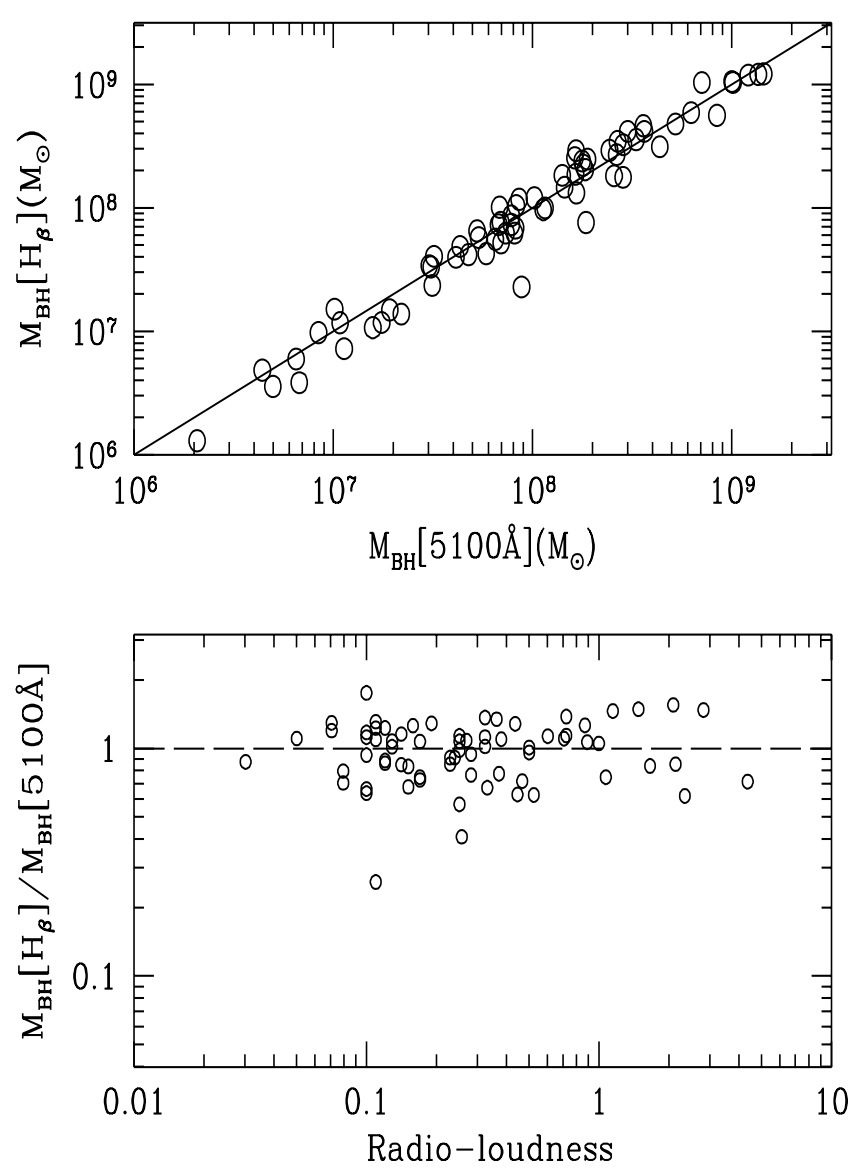

Fig. 4. Upper panel: comparison of black hole masses of 70 radioquiet PG quasars estimated with the $R-L_{\mathrm{H}_{\beta}}$ and $R-L_{5100} \AA$ relations. The diagonal line shows the case where the masses are identical. Lower panel: the ratios of black hole masses estimated with two different relations are plotted against the radio-loudness of radio-quiet quasars. The dashed line indicates that the case where the two black hole mass estimates are identical.

galaxy and spectroscopic measurements of the central velocity dispersions of a large sample of extremely radio-loud quasars are needed to confirm our results.

The advantage of using the $R-L_{\mathrm{H}_{\beta}}$ relation is that we can estimate the black hole mass of AGNs with only two observed parameters, namely the $\mathrm{H}_{\beta}$ line luminosity and its FWHM, and it can be applied to a larger sample of AGNs with redshift smaller than 0.8 . In principle, one can analogously investigate the relation between the BLR size and the luminosity of some ultraviolet emission lines such as MgII and CIV, which may be used to estimate the black hole mass of some high redshift AGNs. Some recent studies have suggested use of the ultraviolet continuum luminosity and the FWHM of ultraviolet emission lines to estimate the black hole mass of high redshift AGNs (Vestergaard 2002; McLure \& Jarvis 2002). However, the ultraviolet continuum luminosity can similarly suffer from serious contamination from the jet and Blamer continuum, therefore the luminosity of the ultraviolet emission line again may be a better indicator of ionizing luminosity than the ultraviolet continuum luminosity.

Finally, one should be cautious of the uncertainties in estimating the black hole mass of AGNs using the $R-L_{\mathrm{H}_{\beta}}$ relation. 
Firstly, variations of $\mathrm{H}_{\beta}$ emission line flux and its FWHM are common in AGNs. Estimating the black hole mass with the values of these two parameters in a single spectrum may lead to large errors. Secondly, the different inclination of the BLR may also significantly affect the results (McLure \& Dunlop 2001; $\mathrm{Wu} \&$ Han 2001). If the BLR has a flattened geometry and the inclination of the BLR is rather small, our derived values of black hole mass may be significantly underestimated. However, the ratio of black hole masses estimated with two empirical relations does not depend on the inclination. Better understanding of the BLR geometry and dynamics is needed to diminish the uncertainties in deriving the black hole mass of AGNs (Krolik 2001).

Acknowledgements. We thank Xinwu Cao, Dongrong Jiang and Ting-Gui Wang for helpful discussions, and the anonymous referee for valuable suggestions which improved the paper significantly. The work is supported by the National Key Project on Fundamental Research (TG 1999075403), the National Natural Science Foundation (No. 10173001) in China and the Jun Zheng Foundation of Peking University. This research has made use of the NASA/IPAC Extragalactic Database (NED) which is operated by the Jet Propulsion Laboratory, California Institute of Technology, under contract with the National Aeronautics and Space Administration.

\section{References}

Boroson, T. A., \& Green, R. F. 1992, ApJS, 80, 109

Brotherton, M. S. 1996, ApJS, 102, 1

Burstein, D., \& Heiles, C. 1982, AJ, 87, 1165

Chiaberge, M., Capetti, A., \& Celotti, A. 2002, New Astron. Rev., 46, 335

Cheung, C. C., Wardle, J. F. C., Chen, T., \& Hariton, S. P. 2003, New Astron. Rev., 47, 423

Collier, S. J., Horne, K., Kaspi, S., et al. 1998, ApJ, 500, 162

Dietrich, M., Peterson, B. M., Albrecht, P., et al. 1998, ApJS, 115, 185

Drinkwater, M. J., Webster, R. L., Francis, P. J., et al. 1997, MNRAS, 284,85
Francis, P. J., Whiting, M. T., \& Webster, R. L. 2000, PASA, 17, 56

Gu, M., Cao, X., \& Jiang, D. R. 2001, MNRAS, 327, 1111

Ho, L. C. 1999, in Observational Evidence for Black Holes in the Universe, ed. S. K. Charkrabarti (Dordrecht: Kluwer), 157

Ho, L. C., \& Peng, C. Y. 2001, ApJ, 555, 650

Isobe, T., Feigelson, E. D., Akritas, M. G., \& Babu, G. J. 1990, ApJ, 364,104

Jester, S. 2003, New Astron. Rev., 47, 427

Kaspi, S., Maoz, D., Netzer, H., et al. 1996, ApJ, 470, 336

Kaspi, S., Smith, P. S., Netzer, H., et al. 2000, ApJ, 533, 631

Lacy, M., Laurent-Muehleisen, S. A., Ridgway, S. E., Becker, R. H., \& White, R. L. 2001, ApJ, 511, L17

Laor, A. 2000, ApJ, 543, L111

Krolik, J. H. 2001, ApJ, 551, 72

Lynden-Bell, D. 1969, Nature, 223, 690

McLure, R. J., \& Dunlop, J. S. 2001, MNRAS, 327, 199

McLure, R. J., \& Jarvis, M. J. 2002, MNRAS, 337, 109

Oshlack, A. Y. K. N., Webster, R. L., \& Whiting, M. T. 2002, ApJ, 576,81

Parma, P., de Ruiter, H. R., Capetti, A., et al. 2003, A\&A, 397, 127

Peterson, B. M., Berlind, P., Bertram, R., \& Bischoff, K. 2002, ApJ, 581, 197

Peterson, B. M., McHardy, I. M., Wilkes, B. J., et al. 2000, ApJ, 542, 161

Peterson, B. M., Wanders, I., Bertram, R., et al. 1998, ApJ, 501, 82

Rees, M. J. 1984, ARA\&A, 22471

Santos-Lleo, M., Chatzichristou, E., Mendes de Oliveira, C., et al. 1997, ApJS, 112, 271

Santos-Lleo, M., Clavel, J., Schulz, B., et al. 2001, A\&A, 369, 57

Scarpa, R., \& Urry, C. M. 2002, New Astron. Rev., 46, 405

Scarpa, R., Urry, C. M., Falomo, R., \& Treves, A. 1999, ApJ, 526, 643

Stirpe, G. M., Winge, C., Altieri, B., et al. 1994, AJ, 425, 609

Vestergaard, M. 2002, ApJ, 571, 733

Wandel, A. 2002, ApJ, 565, 762

Wandel, A., Peterson, B. M., \& Malkan, M. A. 1999, ApJ, 526, 579

Wang, T.-G., \& Zhang, X.-G. 2003, MNRAS, 340, 793

Whiting, M. T., Webster, R. L., \& Francis, P. J. 2001, MNRAS, 323, 718

Winge, C., Peterson, B. M., Horne, K., et al. 1995, ApJ, 445, 680

Wu, X.-B., \& Han, J. L. 2001, ApJ, 561, L59 